\title{
Koiné sículo-toscanizada en los dominios de la Corona de Aragón
}

\author{
Tuscan-Sicilian koine in the dominions \\ of the Crown of Aragón
}

\author{
Salvatore Bartolotta*
}

\begin{abstract}
RESUMEN
En la segunda mitad del siglo XV se advierte un progresivo debilitamiento del siciliano en el uso literario. Folena (1956)

ha indicado la posible influencia, sobre este fenómeno, del italiano de la cancillería aragonesa de Nápoles; la pista debería ser examinada con atención, especialmente en el periodo del rey Alfonso, cuando Nápoles fue el centro de todos los dominios de la corona de Aragón. Sería un error pensar que el italiano hubiera reducido el siciliano a las funciones de simple dialecto. Mientras el siciliano perdía terreno en la esfera

literaria, conservaba, sin embargo, su valor como lengua escrita de la administración; no es fácil decir cuándo se pasó al italiano en este campo, ya que sería necesario distinguir una localidad de

otra. Las fases de este proceso se observan en la tradición manuscrita siciliana del siglo $X V$.
\end{abstract}

\section{PALABRAS CLAVE}

Interlingüística, latín, siciliano, sociolinguistica, toscano.

\section{ABSTRACT}

In the second half of the 15th century a progressive weakening of the Sicilian language in the literary use is noticed. Folena (1956) has pointed out the possible influence of the Italian language of the Aragonese chancellery of Naples on this phenomenon; the clues would have to be examined with attention, specially during King Alfonso's period, when Naples was the center of all the dominions of the Crown of Aragón. It would be an error to think that the Italian language had reduced the Sicilian language to the functions of a mere dialect. While the Sicilian language lost ground in the literary sphere, it preserved, however, its value as the written language of the administration; it is not easy to say when the Italian language started to be used in this field, since it would be necessary to distinguish different features depending on the place. The phases of this process can be seen in the Sicilian handwritten tradition of the 15th century.

\section{KEY WORDS}

Interlinguistics, Latin, Sicilian, sociolinguistics, Tuscan.

* Departamento de Filologías Extranjeras y sus Lingüísticas. UNED. 
En el siglo XIV e inicios del XV la influencia del toscano se hace bastante notable en Sicilia, por lo que algunas obras escritas en siciliano se traducen al toscano. Folena (1956: LV-LVI) escribía hace algunos años lo siguiente sobre el Eneas:

Di fronte al toscano il siciliano è ancora una «lingua» straniera: i toscanismi sono ancora occasionali [...] si tratta in massima parte di episodi lessicali, e assai più importanti come strumento e modello di elaborazione letteraria, è tuttora il latino. Quando un tipo fonetico toscano si oppone a un tipo siciliano, esso è nella maggioranza dei casi in simbiosi col tipo latino'.

En la isla, como en otros lugares, el prestigio del toscano se debe a la poesía, sobre todo a la de Petrarca. Los petrarquistas sicilianos del siglo XV son conocidos a través de las menciones y citas de Mario De Arezzo en las Osservantii di la lingua siciliana (1543); nada más sabemos de Bartolomé Corbera, Andria Claramonte y los demás, que parece que hayan usado «un siciliano fuertemente toscanizado antes, y probablemente el toscano después»². Completamente marginados aparecen algunos episodios de alternancia lingüística, como los de la comedia de Cayo Caloria Ponzio, estudiante en Padua ${ }^{3}$; ni siquiera incide en modo específico en la historia lingüística de Sicilia el latín de los humanistas, que refuerza el uso de esta variedad en algunos dominios de la lengua escrita sin debilitar directamente al vulgar como sucede en otros lugares.

Es más importante, en cambio, que ya en la segunda mitad del siglo XV se advierta un progresivo debilitamiento del siciliano en el uso literario. Folena ha indicado 4 la posible influencia, sobre este fenómeno, del italiano de la cancillería aragonesa de Nápoles; la pista debería ser examinada con atención, especialmente en el periodo del rey Alfonso, cuando Nápoles fue el centro de todos los dominios de la corona de Aragón. Pero el italiano se revela a nivel literario mucho antes que a nivel burocrático. Las fases de este proceso se observan en la tradición manuscrita de las obras sicilianas del siglo XV: el manuscrito $B$ del Eneas, mesinés de finales del siglo XV, toscaniza fuertemente la lengua de la obra de modo que Folena ${ }^{5}$ duda entre definirla como toscana con fuertes residuos sicilianos o una koiné sículo-toscanizada. También significativo es el caso de la Leggenda de la Beata Eustachia (1478), que M. Catalano considera escrita en «una lengua toscana modelada sobre el latín y constituida por frecuentes incidencias sicilianas» ${ }^{6}$. Esta obra también es

1 «Frente al toscano el siciliano resulta todavía una lengua extranjera: los toscanismos son aún ocasionales [...] se trata en buena parte de fenómenos léxicos aislados, y aún más importante, como instrumento y modelo de elaboración literaria, sigue siendo el latín. Cuando un tipo fonético toscano se opone a un tipo siciliano, el mismo se halla en la mayoría de los casos en simbiosis con el tipo latino".

2 Cfr. Cusimano (1951: 14).

3 Cfr. Rossi (1930: 417-451).

4 Cfr. Folena (1956: LXIII).

5 Ibid., LXI.

6 Cfr. Catalano (1950: 33). Nótese todavía que los manuscritos de la leyenda no son de copistas sicilianos. 
mesinesa y es verosímil que Messina anticipe el periodo de la toscanización respecto a las otras ciudades de la isla ${ }^{7}$ : por ello el mismo fenómeno se encuentra en las ediciones incunables ${ }^{8}$ mesinesas, que comienzan justo en 1478 . Folena indica ${ }^{9}$ como análoga a la del manuscrito B del Eneas la lengua de la Protesta dei Messinesi (1478) y lo mismo se puede decir de la Scala de virtute $(1499)^{10}$.

Sería un error pensar que el italiano hubiera relegado al siciliano a las funciones de simple dialecto. Mientras el siciliano perdía terreno a nivel literario, conservaba, sin embargo, su valor como lengua escrita de la administración; no es fácil decir cuándo se pasó al italiano en este campo, ya que sería necesario analizar su uso por localidades. Es verdad que, por ejemplo, cuando el 4 de agosto de 1507 el notario de Monreal lleva a cabo el inventario del tesoro de la catedral, a petición del gobernador de la ciudad, lo hace en siciliano ${ }^{11}$. Mucho más tarde, en 1537, el inventario del castillo de Castellamare del Golfo se escribe en siciliano y no en italiano o latín ${ }^{12}$. En algunos documentos del Despacho de Catania el siciliano sigue siendo usado en 1572 y $1583^{13}$ y lo mismo pasa en los inventarios del tesoro de S. Águeda, en la misma ciudad hasta 1556'14; además todavía en 1556 aparece en siciliano la autorización por parte del virrey Vega para la fundación del colegio de los Jesuitas en Catania $^{15}$. Según estas indicaciones, la vitalidad del siciliano burocrático ha sido bastante larga y no puede considerarse fácil la victoria del italiano.

Examinando un grupo de cartas de los años veinte del siglo XVI, se observa ${ }^{16}$ que el uso del italiano parece obedecer, al inicio, a criterios completamente personales; se trata de cartas enviadas a la localidad de Buscemi (hoy en provincia de Siracusa) desde Giarratana, Vizzini, Monterojo y Módica ${ }^{17}$, siempre refiriéndose al ganado capturado en las tierras de Buscemi. Las cartas están escritas en siciliano, como era normal en ese periodo, excepto la de los jurados de Monterojo, que tiene el marco en latín y el texto en italiano con escasos errores. El examen de las varias posibilidades de explicación de la anomalía muestra que la misma debe ser atribuida a la iniciativa individual de quien escribe, de seguro sugestionado, en el pequeño centro en que se encuentra, por una moda cultural propagada por la imprenta.

7 Obsérvese todavía que Migliorini (1988: 281), erra en referir a Messina los protocolos notariales que se italianizarían a partir de 1492: la noticia proviene de Catalano (1950: 39, cita 33), pero se refiere a Catania.

8 Según DRAE 2001, incunable (del lat. incunabula 'pañales') se refiere a las ediciones hechas desde la invención de la imprenta hasta principio del siglo XVI.

9 Cfr. Folena (1956: LXI).

10 Cfr. por el momento Catalano (1950: 42).

11 Cfr. ed. G. Millunzi, en Archivio Storico Siciliano XXVIII (1903: 305-316).

12 Cfr. ed. P. M. Rocca, en Archivio Storico Siciliano X (1885: 324-329).

13 Cfr. G. Scalia, en Archivio Storico per la Sicilia Orientale XXX (1934: 181-234).

14 Cfr. C. Musumarra, en Archivio Storico per la Sicilia Orientale XLVIII (1952: 39-111) y cfr. ibid. XXI, (1925: 33) y ss.

15 Cfr. M. Catalano, en Archivio Storico per la Sicilia Orientale XIV (1917: 168).

16 Cfr. Vàrvaro (1977: 3-5).

17 Editadas por G. Verdirame, en Archivio Storico per la Sicilia Orientale III (1906: 75-77). 
Así, ya en el segundo cuarto del siglo XVI el italiano se extiende a los rincones más apartados de la isla. Después de cincuenta años es el italiano la lengua dominante: los jurados de Catania escriben alrededor de treinta cartas a Don Juan de Austria ${ }^{18}$ y se sirven siempre del italiano; sólo en la carta dirigida a un privado el siciliano aparece sometido a la preponderancia del italiano. En 1571-1572 el italiano es definitivamente la lengua escrita utilizada por la burocracia de la isla, con alguna excepción justificada por el destinatario (siciliano e íntimo) y por el argumento (también familiar). Se trata lógicamente de un italiano poco depurado de elementos locales y excesivas hipercorrecciones: entre los primeros se recuerda potiva, stisa, dassiro, presentao, el uso del imperfecto subjuntivo por el condicional, términos como scasare, lettra, formento; entre las segundas alconi, piò, nui ${ }^{19}$. Pero de todos modos se trata sin duda del italiano.

Desde que la imprenta, la literatura escrita y hasta la burocracia lo difundieron ampliamente, el italiano ve reconocida su validez en las discusiones de los intelectuales estudiadas por Sorrento (1921) ${ }^{20}$. Todavía en 1593 De Arezzo defendía el sículo - toscano de los petrarquistas del siglo XV, pero a mediados de dicho siglo se admite la supremacía del italiano, ya considerada en la práctica y confirmada en la segunda mitad del siglo por la aparición de obras literarias en italiano, comenzando por las de A. Giuffredi y P. Caggio en prosa y por los poemas heroicos y caballerescos en verso. Pero todavía más significativo es que aparezca una memorialística, a veces de intención casi privada, que adopta también el italiano. Un ejemplo extremadamente precoz es la Cronica de un tal Antonio Merlino de quien sólo sabemos que probablemente era un catanés, que narra los acontecimientos de 1516, todavía recientes ${ }^{21}$. En Merlino, por ejemplo, las vocales finales sicilianas son menos frecuentes respecto a las italianas. El uso del italiano es la norma de la diarística de finales del siglo, además de la del siglo XVII22. Pero no se debe dejar de lado la circunstancia de que este italiano escrito semi-privado esté en continuo contraste con el siciliano escrito del siglo anterior: mientras este último presentaba un léxico latinizado o escasez de palabras del uso vivo, por el contrario el italiano está fuertemente marcado por el florecimiento de términos del uso vivo y a veces de giros sintácticos locales. Frente al siciliano escrito que refleja una norma definida y escasa relación con la lengua hablada, la norma italiana aparece mal dominada y por lo tanto a veces violada involuntariamente. Si antes la lengua escrita mantenía cierta relación orgánica con la hablada y por lo tanto se destacaba con plena conciencia, ahora en cambio es una lengua contenida en los libros y que encuentra su norma en la gramática y en los modelos escritos provenientes de otras regiones, sin que se alcance la elasticidad necesaria para responder adecuada-

18 Editadas por B. Vaccalluzzo Fagioli, en Archivio Storico per la Sicilia Orientale VI (1909: 1-19); estudiadas en Vàrvaro (1977: 5).

19 Cfr. Vàrvaro (1977: 5).

20 Cfr. también la contribución de Beretta Spampinato (1980: 361-85).

21 Editada por G. Salvo Cozzo, en Archivio Storico Siciliano VI (1881: 112-29).

22 Cfr. Di Marzo (1869-86). 
mente a todos los problemas planteados por la escritura y en particular sin ductilidad sintáctica y sin recursos léxicos espontáneos: si el modelo pierde valor, quien escribe no puede hacer más que recurrir al término más conocido para él, que es obviamente el dialectal.

Algún ensayo nos puede dar una idea completa de lo que era, en el siglo XVI, el italiano escrito de Sicilia. En un nivel más alto, que sólo pocos lograban dominar, podemos encontrar una lengua bastante cuidada, no muy diferente del italiano de las otras regiones, a veces también vivaz y consistente, como en la carta sarcástica que Juan Guillermo Bonascontro o Bonincontro -encarcelado por la Inquisiciónescribió en 1568 al obispo de Cefalù, mezclando el italiano con el latín. El autor afirma conocer «linguam latinam, germanicam, gallicam, hispanam, ianuensem, venetam, bergamascam, florentinam, neapolitanam, siculam et bergariotam " (la última será probablemente el briariotu hablado en la popular barriada de la Alberguería de Palermo), de modo que pueda ser adecuada «para la hostería V. S. R.ma, nuevamente abierta en Cefalù con el dulce, amado y solicitado aviso: Propinasti gratis» (se trata de una nueva sección del Tribunal de la Inquisición):

Perché, oltre che riceverei ciascheduno forestiero allegramente e con bona cera alla bolognese, dando unicuique merita sua et parlando in un suo linguaggio, saprei come vecchio cortegiano che sono, conversare con spagnuoli: cosa veramente difficile assai che andar per via ordinaria! Perché ci vuol altro che baie a voler stare con la misura, co'l compasso, co'l peso e co'l lidio lapide in mano tutte le hore per misurare, compassare, pesare, squadrare le longitudini, le latitudini e le profondità de' menti de ciascheduno d'essi, et havere ogni momento l'hostia in bocca per tema di commettere alcuni errori con fatti loro; considerare a cui tocca seggia di velluto, a cui di cuoio, e qui viene un'altra maledetta distinzione, cioè a cui la regale e a cui la bastarda; item a cui tocca brocchetta e a cui tocca sottocoppa et a cui non; item a cui si ha da parlar di V. S., a cui di V. Magnificenza, a cui di vos et a cui di tu; col mal'anno e la mala Pasqua che Dio dia loro e a chi tanti ne porta a questi nostri paesi! ${ }^{23}$.

En este fragmento hay poco de siciliano (también seggia y a cui pueden justificarse) y se descuidan las incertidumbres (ciascheduno por ciaschedun y bona por buona, además de de' menti, si no es un error del transcriptor). Menor dominio demuestra la extensión de un memorial sobre la larga causa que opuso al fisco de la

${ }^{23}$ Cito según la edición de C. A. Garufi, en Archivio Storico Siciliano XL (1915: 362). «Porque, además de recibir a cada uno de los forasteros alegremente con buena cara a la boloñesa, dando unicuique merita sua y hablando su propia lengua, sabría como viejo cortesano que soy, conversar con españoles: jalgo más difícil todavía que andar por vía ordinaria! Porque se necesita más que tener cuidado para querer estar en la medida, con el compás, con el peso y con el lápiz en la mano todo el tiempo para medir, compasar, pesar y regular las longitudes, las latitudes y las profundidades de la mente de cada uno de ellos, y tener en cada momento la hostia en la boca para evitar cometer errores con sus asuntos; considerar a quien le toca la silla de terciopelo, a quien la de cuero, y aquí se hace otra maldita distinción, es decir a quien la real y a quien la bastarda; lo mismo a quien le toca una jícara, a quien una salvilla y a quien no; también en cuanto a quien tiene que hablar de V. S., a quien de V. Magnificencia, a quien de vos y a quien de tu; ;con el mal año y la mala Pascua que Dios les de a ellos y a quienes llevan a estos países nuestros!». 
Inquisición al mismo Bonincontro para la herencia de su tío Ángel Panicola. He aquí un párrafo:

Item voli provari che un giorno di dicto anno lo dicto magn. Dovico ${ }^{24}$ andao in casa di dicto Giovanni Guglielmo suo frati, in lo muraturi undi solia stari prima, a lo canto di notar Petro di Ricca, et li dissi questi o simili palori: «Signor Giovanni Guglielmo, io vi ripudio et refutu lu locu della Turri vechia, chi mi lassao nostro zio Ambroso Panicula, et voglio chi lu habiati vui, zoè heredi universali che siti, perché s'io lu accepto et piglo possessioni, subito mi verranno a dosso li subiugatarii et io non hagio forma di pagarli, né manco di teneri boi et homeni per gubernari lo dicto locu. Mandati a chiamari a un notaru che io vinni faczo un acto in larga forma ${ }^{25}$.

Además de las huellas del latín jurídico y las deficiencias de la forma sintáctica general, y además términos como vechia, habiati y piglo, nótese la fuerte incidencia del siciliano en el vocalismo tónico (muraturi, solia, turri, vui, siti; también la falta de diptongo en voli, locu, boi, homeni es significativa) y átono (en el interior de palabras como muraturi y gubernari, además del hipercorrecto homeni, pero sobre todo en las numerosas vocales finales -i y - u), en la consonante de zoè y de fazzo, en la metátesis de palori, en las formas lu, lo por il, li por gli, vinni por ve ne, en los verbos andao, lassao, hagio, en el acusativo personal con a (en chiamari a un notaru), en palabras como frati, muraturi, undi y canto.

Está claro que el italiano escrito en Sicilia sufre una influencia de dialectalismos que se relaciona tanto con el nivel de instrucción y cultura de quien lo escribe como con la fecha (en el sentido que la incidencia decrece ligeramente con el pasar del tiempo), como, además, con el argumento. Los diaristas, por ejemplo, resultan muy sensibles a esta última variación: si su lengua es relativamente correcta cuando se refiere al virrey y las ceremonias oficiales, la cosa cambia cuando se habla de episodios cotidianos. He aquí como Vicente Auria describe un delito acaecido en 1606:

Fu ammazzatu con una scopettata il padre fra Isidoro di Trapani carmelitano, predicatore famosissimo, affacciandosi alla fenestra della casa di sua sorella, dentro la vanella dietro il piano delli Bologni26.

\footnotetext{
24 Se refiere a Ludovico Bonincontro, hermano de Juan Guillermo, éste último ya nombrado anteriormente a propósito de la carta sarcástica que éste escribió en 1568 al obispo de Cefalù.

${ }_{25}$ El párrafo forma parte del mismo trabajo de C. A. Garufi, ibid., p. 367. «Lo mismo quiere probar que un día de dicho año el llamado magnífico Dovico fue a la casa de dicho hermano suyo Juan Guillermo, en la morada donde frecuentemente estaba antes, junto al notario Pedro de Rica, y le dijo estas o parecidas palabras: "Señor Juan Guillermo, yo rechazo y reniego el lugar de la Torre vieja, que me dejó nuestro tío Ambrosio Panicula, y quiero que lo tenga usted, como heredero universal que es, porque si yo lo acepto y tomo posesión, inmediatamente me atacarán malefactores y yo no tengo forma de pagarles ni siquiera tengo bueyes ni hombres para sostener dicho lugar. Mande a llamar a un notario que yo vengo y hago un acta en la mejor forma"».

${ }_{26}$ Cfr. Di Marzo (1869-1886: I: 222). «El padre fray Isidoro de Trapani —carmelita, predicador famosísimo- fue asesinado con una escopeta mientras se asomaba a la ventana de la casa de su hermana, en la calle detrás del barrio de los Boloñi».
} 
Además del uso bastante libre del gerundio, típico de estos diarios (aquí affacciandosi vale por 'mientras se asomaba'), a la hipercorrección fenestra, al delli, el léxico es el de la expresión hablada: ammazzare, scopettata, vanella, piano. Todavía más evidente es el fenómeno donde Baltasar Zamparrone refiere acontecimientos de 1603:

Essendo costituito il loco del mercato in lo piano della Marina, come ho detto, fra l'altro cose che vennero vi fôro certe pira moscarelle di Bivona, quale bvendia lo stesso che li portò, et quando pesava faceva bonissimo peso. II che vedendo un potigaro, s'accostò e li disse che, se li dava tarì quattro, esso ci voleva pisari li pira et fare grand'utile, e dare unzi 9 per rotolo. II che avendo inteso l'officiali della città, lo presero; e il pretore ordinò che posse posto alla vergogna. $E$ così fu posto alla cantonera della Vicarìa, sotto la tortura, legato con ferri a li pedi, scappellato. Dapoi fôro pigliati due gallinari seu rigatteri, che compravano pullami per rivenderla, et fôro anco posti in la virgogna ${ }^{27}$.

Aquí también el vocalismo aparece a veces incierto (loco, pira, bonissimo, pedi, pisari, unzi, pullami, virgogna), las desinencias morfológicas son a veces sicilianas (pira, unzi, pullami fem. sing.), artículos y pronombres personales tienen forma dialectal (alli, li, lo, li 'el', ci 'los'), quale funciona como 'que', términos como piano, potigaro, cantonera, scapellato, rigatteri son semánticamente y a veces también formalmente del siciliano.

De hecho, este siciliano escrito pero no hablado mantiene -en sus formas más modestas y privadas - una relación fecunda con el dialecto, hasta el punto de no constituir la lengua muerta que a menudo aparece a nivel literario.

\section{REFERENCIAS BIBLIOGRÁFICAS}

ALFIERI, G. (1992): «La Sicilia», F. Bruni (ed.), L'italiano nelle regioni. Lingua nazionale e identità regionali, Torino: UTET, 798-860.

AmbrosinI, R. (1977): Stratigrafia lessicale di testi siciliani dei secoli XIV e XV, Palermo: Centro di Studi Filologici e Linguistici Siciliani.

Bartolotta, S. (2000): Perfil de historia lingüística de Sicilia en la edad aragonesa y castellana, trabajo de investigación, Madrid: UNED.

- (2004): «Breve recorrido histórico-lingüístico en la Sicilia de la Guerra de las Vísperas», Espacio, forma y tiempo, Serie III, 17, 67-78.

- (2005A): II dialetto di Castrofilippo, Bari: Laterza.

- (2005B): «Las interferencias entre el siciliano y el latín en el siglo XIV», J. Costa Rodriguez (ed.), Ad amicam amicissime scripta, Madrid: UNED, II, 201-08.

27 Ibid., p. 264. «Siendo constituido el lugar del mercado en el barrio de la Marina, como he dicho, entre otras cosas que pasaron fueron ciertas peras moscarelas de Bivona, que vendía el mismo que las llevó, y cuando las pesaba hacía un buen peso. Viendo esto un bodeguero, se acercó y le dijo que si le daba cuatro tarines, él le pesaba las peras y hacía una buena obra, dándole 9 onzas por rótulo. Por lo que escuchando eso unos oficiales de la ciudad, lo detuvieron; y el pretor ordenó que se castigara la vergüenza. Y así fue conducido al rincón de la Vicaría, bajo la tortura, atado con hierros en los pies, y golpeado en la cabeza. Luego capturaron a dos gallineros o ropavejeros, que compraban pollos para revenderlos, y fueron también castigados por la vergüenza». 
- (2006A): «Interferencia catalano-siciliana en el Reino de Aragón», M. Villayandre Llamazares (ed.), Actas del XXXV Simposio Internacional de la Sociedad Española de Lingüística, León, 12-15 de diciembre de 2005, Universidad de León, Dpto. Filología Hispánica y Clásica, 211-220.

- (2006B): «Fenómenos de interferencia en la Sicilia no románica», Interlingüística, 17, 175-182.

Bartolotta, S. Lunetta, S. Messina, C. Tedeschi, P. y Virgillitto, S. (1993): “Confessionale Il»: Analisi linguistica e confronto con "Libru di lu transitu et vita di messer Sanctu Iheronium» ed "Ordini di la confessioni Renovamini», trabajo de investigación, Catania: Università degli Studi di Catania.

BATTAGLIA, S. (1962-2002): Grande dizionario della lingua italiana, 21 vols., Torino: UTET.

BECCARIA, G. L. (1968): Spagnolo e spagnoli in Italia, Torino: Giappichelli.

Beretta Spampinato, M. (1980): «La prosa del '500", Storia della Sicilia, IV, 361-85.

BrEsc, H. (1971): Livre et société en Sicile (1299-1499), Palermo: Centro di Studi Filologici e Linguistici Siciliani.

- (1986): Une monde méditerranéen. Economie et société en Sicile. 1300-1450, Roma - Palermo: Ecole Française de Rome - Accademia di Scienze, Lettere e Arti di Palermo.

BrEsc, H. y GoITEIN S. D. (1970): «Un inventaire dotal des juifs siciliens (1479)», Mélanges d'archéologie et d'histore de l'Ecole française de Rome, 82, 903-17.

BRuNI, F. (1980): «La cultura e la prosa volgare nel '300 e nel '400», Storia della Sicilia, IV, 179-278.

- (ed.) (1992): L'italiano nelle regioni. Lingua nazionale e identità regionali, Torino: UTET.

Catalano, M. (1950): La leggenda della Beata Eustochia da Messina, Messina - Firenze: D'Anna.

Corrao, P. (1991): Governare un regno. Potere, società e istituzioni in Sicilia tra Trecento e Quattrocento, Napoli: Liguori.

CusIMANO, G. (ed.) (1951): «Poesie siciliane dei secoli XIV e XV», Collezione di testi siciliani dei secoli XIV e $X V, 3$, Palermo.

D’Agostino, G. (1979): Parlamento e società nel regno di Napoli. Secoli XV - XVII, Napoli: Guida.

D'Alessandro, V. (1963): Politica e società nella Sicilia aragonese, Palermo: Manfredi.

- (1986): «Il Mezzogiorno dagli Angioini agli Aragonesi», La storia. I grandi problemi dal Medioevo all'Età contemporanea, II, 523-533.

Del Treppo, M. (1972): I mercanti catalani e l'espansione della corona aragonese nel secolo XV, Napoli: Collana del Seminario di Storia medioevale e moderna dell'Università di Napoli, 4.

- (1986): «ll regno aragonese», G. Galasso y R. Romeo (eds.), Storia del Mezzogiorno, IV, Roma - Napoli: Edizioni del Sole, 89-201.

DI MARZO, G. (1869-1886): «Diari della città di Palermo dal secolo XVI al XIX», Biblioteca Storica e Letteraria di Sicilia, Palermo.

DRAE (2001): Diccionario de la lengua de la Real Academia Española, 2 vols., XXII ed., Madrid: Espasa Calpe.

FolENA, G. (ed.) (1956): «La istoria di Eneas vulgarizata per Angilu di Capua», Collezione di testi siciliani dei secoli XIV e XV, 7, Palermo.

GalAsso, G. (1992): // Regno di Napoli. I/ Mezzogiorno angioino e aragonese (1266-1494), Torino: UTET.

Galasso, G. y Romeo, R. (eds.) (1986): Storia del Mezzogiorno, vol. IV, Roma - Napoli: Edizioni del Sole.

GIUNTA, F. (1973): «Un gruppo di lettere in siciliano di Eleonora d'Aragona (1375)», Bollettino del Centro di Studi Filologici e Linguistici Siciliani, 12, 346-53.

Holtus, G. (1989): «Per una carta dialettologica italiana», en G. Holtus, M. Metzeltin y M. Pfister (eds.), La dialettologia italiana oggi. Studi offerti a Manlio Cortelazzo, Tübingen: Narr, XXXII y LIII-LVI.

Holtus, G. Metzeltin M. y PFISTER M. (eds.) (1989): La dialettologia italiana oggi. Studi offerti a Manlio Cortelazzo, Tübingen: Narr.

LeONE, A. (ed.) (1990): Il vocabolario siciliano - latino di Lucio Cristoforo Scobar, Palermo: Centro di Studi Filologici e Linguistici Siciliani.

LI GotTI, E. (ed.) (1951): Volgare nostro siculo. Crestomazia di testi siciliani del sec. XIV, I, Firenze: La Nuova Italia.

MATTESINI, E. (1994): «Sicilia», L. Serianni y P.Trifone (eds.), Storia della lingua italiana, Torino: Einaudi, III, 406-432.

MigLIORINI, B. (1988): Storia della lingua italiana, 2 vols., Firenze: Sansoni (reim. con la introducción de G. Ghinassi).

MoccIARO, A. G. (1975): «Vocabolari siciliani manoscritti inediti», La ricerca dialettale, 1, 423-444.

NALLI, P. (1938): «Saggio bibliografico sui testi a stampa in dialetto siciliano», Scritti vari dedicati a Mario Armanni in occasione del suo sessantesimo compleanno, Milano: Hoepli, 159-98.

PAgliaro, A. (ed.) (1953): Saggi di critica semantica, Messina - Firenze: D'Anna.

- (ed.) (1956): Nuovi saggi di critica semantica, Messina - Firenze: D'Anna. 
Pellegrini, G. B. (1977): Carta dei dialetto d'Italia, Pisa: Pacini.

PIccitTo, G. (1950): «La classificazione delle parlate siciliane e la metafonesi in Sicilia», Archivio storico per la Sicilia orientale, serie IV, III, 5-34.

PITRĖ, G. (1928): «Supplemento ai dizionari siciliani», Studi Glottologici Italiani, VIII, 1-119.

QuATtordio MoreschinI, A. (ed.) (1984): Tre millenni di storia linguistica della Sicilia (Atti del Convegno della Società Italiana di Glottologia, Palermo 25-27 marzo 1983), Pisa: Giardini.

RoHLFS, G. (1975): Historische Sprachschichten in modernen Sizilien, München: Bayerischen Akademie d. Wissenschaft (tr. it. La Sicilia nei secoli, Palermo: Sellerio, 1984).

- (1977): Supplemento ai vocabolari siciliani, München: Bayerischen Akademie d. Wissenschaft.

ROMANO, D. (1973): «Un testo in volgare siciliano del 1351», Culture regionali e letteratura nazionale (Atti del VII Congresso dell'Associazione Internazionale per gli Studi di Lingua e Letteratura Italiana, Bari 31 marzo-4 aprile 1970), Bari: Adriatica Editrice, 387-391.

Rossı, V. (1930): “Caio Caloria Ponzio e la poesia volgare letteraria di Sicilia del secolo XV», Scritti di critica letteraria, II, Firenze.

RufFINO, G. (1984): «Isoglosse siciliane», A. Quattordio Moreschini (ed.), Tre millenni di storia linguistica della Sicilia (Atti del Convegno della Società Italiana di Glottologia, Palermo 25-27 marzo 1983), Pisa: Giardini, 161-224.

SERIANNI, L. y TRIFONE P. (eds.) (1994): Storia della lingua italiana, 3 vols., Torino: Einaudi.

SORRENTO, L. (1921): La diffusione della lingua italiana nel Cinquecento in Sicilia, Firenze: Università degli Studi di Firenze - Biblioteca di Scienze Sociali.

StarRabba, R. (1873): «Processo di fellonia contro frate Simone Del Pozzo vescovo di Catania (1392)», Archivio Storico Siciliano, I, 401-402.

- (ed.) (1887-1888): Lettere e documenti relativi a un periodo del vicariato della Regina Bianca in Sicilia (1411-1412), Palermo: Società Italiana per la Storia Patria (reimp. 1993).

TrainA, A. (1868): Nuovo vocabolario siciliano - italiano, Palermo: Edizioni Sedilis (reimp. 1977).

- (1877-88): Vocabolarietto delle voci siciliane dissimili dalle italiane, Palermo: Libr. Intern. L. PedoneLauriel di C. Clausen.

TramontanA, S. (1989): Gli anni del Vespro, Bari: Dedalo.

VÀRVARO, A. (1974): «Prima ricognizione dei catalanismi nel dialetto siciliano», Medioevo romanzo, I, 86110.

- (1977): «Note per la storia degli usi linguistici in Sicilia», Lingua Nostra, XXXVIII, 1-7.

- (1979): Profilo di storia linguistica della Sicilia, Palermo: Flaccovio.

- (1981): Lingua e storia in Sicilia. Dalle guerre puniche alla conquista normanna, Palermo: Sellerio.

- (1984): La parola nel tempo. Lingua, società e storia, Bologna: Il Mulino.

- (1988): «Aree linguistiche, XII. Sicilia», Lexikon der Romanischen Linguistik, 4, 716-731.

Vitolo, G. (1986): II regno angioino, G. Galasso y R. Romeo (eds.), Storia del Mezzogiorno, IV, Roma Napoli: Edizioni del Sole, 11-86.

Vitolo, G. (1994): Corso di Storia I. Medioevo, Milano: Bompiani.

VS (1977-2002): Vocabolario siciliano, Vol. I (A-E), G. Piccitto (ed.), Catania - Palermo: Centro di Studi Filologici e Linguistici Siciliani, 1977; Vol. II (F-M), G. Tropea (ed.), Catania - Palermo: Centro di Studi Filologici e Linguistici Siciliani, 1985; Vol. III (N-Q), G. Tropea (ed.), Catania - Palermo: Centro di Studi Filologici e Linguistici Siciliani, 1990; Vol. IV (R-S), G. Tropea (ed.), Catania - Palermo: Centro di Studi Filologici e Linguistici Siciliani, 1997; Vol. V (Si-Z), S. C. Trovato (ed.), Catania - Palermo: Centro di Studi Filologici e Linguistici Siciliani, 2002. 